\title{
Investigation into the optimal prosthetic material for wound healing of abdominal wall defects
}

\author{
ADEM AKCAKAYA ${ }^{1}$, IBRAHIM AYDOGDU ${ }^{2}$ and BULENT CITGEZ ${ }^{3}$ \\ Departments of ${ }^{1}$ General Surgery and ${ }^{2}$ Pediatric Surgery, Bezmialem Vakif University, \\ Faculty of Medicine, 34093 Istanbul; ${ }^{3}$ Department of General Surgery, Sisli Hamidiye Etfal Training \\ and Research Hospital, Faculty of Medicine, 34371 Istanbul, Turkey
}

Received May 29, 2017; Accepted November 22, 2017

DOI: $10.3892 /$ etm.2017.5551

\begin{abstract}
The purpose of this experimental study is to investigate and compare the effects of prosthetic materials used for wound healing of abdominal wall hernias. A total of 60 rats were divided into five equal groups: Group I, control subjected to laparotomy; group II, abdominal wall defect $3 \times 2 \mathrm{~cm}+$ polypropylene (PP) mesh; group III, abdominal wall defect $3 \times 2 \mathrm{~cm}+\mathrm{PP}$ mesh+hyaluronate and carboxymethylcellulose (H-CMC; Seprafilm ${ }^{\circledR}$ ); group IV, abdominal wall defect $3 \times 2 \mathrm{~cm}+$ polytetrafluoroethylene (PTFE; Composix ${ }^{\mathrm{TM}}$ ); and group $\mathrm{V}$, abdominal wall defect $3 \times 2 \mathrm{~cm}+$ polyethylene terephthalate (PET; Dacron ${ }^{\circledR}$ ). A total of 14 days after the surgery, rats were sacrificed and the meshes with the surrounding tissue were extracted in block. The breaking strength of the mesh from the fascia was recorded. The healing tissue was examined with the index of histopathology and the hydroxyproline value was analyzed using the Switzer method. Both the breaking strength and histopathological index of the wound healing were significantly improved in groups II and III compared with that in groups IV and V $(\mathrm{P}<0.001)$. Hydroxyproline values were the highest in group $\mathrm{I}(\mathrm{P}<0.001)$. There was also a statistically significant difference between groups II and IV, and group $\mathrm{V}$ and the other groups $(\mathrm{P}<0.001)$. The present findings demonstrated that PP mesh and PP mesh+H-CMC had a superior breaking strength and improved histopathologic indices compared with PTFE and PET. Furthermore, hydroxyproline values were the lowest in the PET group. In conclusion, wound healing was improved in the PP mesh group and the PP mesh+H-CMC group compared with the PTFE and PET groups according to the present study parameters.
\end{abstract}

Correspondence to: Dr Ibrahim Aydogdu, Department of Pediatric Surgery, Bezmialem Vakıf University, Faculty of Medicine, Adnan Menderes Boulevard, 2 Vatan Street, Fatih 34093 Istanbul, Turkey E-mail: draydogdu@yahoo.com

Key words: wound healing, incisional hernia, mesh, prosthetic material

\section{Introduction}

The incidence of incisional hernia is approximately 10 to $50 \%$ after abdominal surgery, and the risk for wound infection increases markedly (1). Incisional hernias are repaired with primer sutures or prosthetic mesh (2). The recurrence rate after primary repair is up to $31 \%$ (3). In recent studies, it has been found that recurrent hernia repair with prosthetic mesh is much less frequent (2).

Incisional hernias quite often appear with difficulties in surgery clinics. Hernia repair treatment has improved recently, in parallel with the development in the technological era (4). Synthetic materials increase the tissue durability while used in abdominal hernias but on the other hand these materials cause intra-abdominal adhesions especially in the wide defects (5).

In our current practice, we most frequently use PP based meshes. Dual meshes have been widely preferred due to improvement in laparoscopic surgery, recently. It is used to prevent the recurrence which is the most important consideration, but avoiding intra-abdominal adhesions has reached the first priority nowadays. We aimed to investigate the effects of different prosthetic materials in the repair of abdominal wall defect on wound healing by measuring the hydroxyproline level, histopathologic index and breaking strength.

\section{Materials and methods}

This study was performed at The Institute of Experimental Medicine, Istanbul University. The study protocol was approved by the Institutional Animal Care and Use Ethics Committee at Istanbul University. A total of 60 adult, female and Wistar albino rats weighing 202-254 g were used as experimental animals. The animals were divided into five equal groups: Group I, Control, Laparotomy; group II, abdominal defect $(3 \times 2 \mathrm{~cm})+$ polypropylene (PP) mesh; group III, abdominal defect $(3 \times 2 \mathrm{~cm})+\mathrm{PP}$ mesh+hyaluronate and carboxymethylcellulose (H-CMC; Seprafilm $\left.{ }^{\circledR}\right)$; group IV, abdominal defect $(3 \times 2 \mathrm{~cm})$ + polytetrafluoroethylene (PTFE; Composix ${ }^{\mathrm{TM}}$ ); and group V, abdominal defect $(3 \times 2 \mathrm{~cm})+$ polyethylene terephthalate (PET; Dacron $\left.^{\circledR}\right)$.

The animals were housed at $21^{\circ} \mathrm{C}$ and were given tap water and standard rat food. After one night of fasting, animals were anesthetized by an intramuscular injection of ketamine 
hydrochloride (Ketalar ${ }^{\circledR}$; Pfizer, Inc., New York, NY, USA; 50-100 mg per kg of body weight). Laparotomy was performed with a $3 \mathrm{~cm}$ incision made in the middle line to create $3 \times 2 \mathrm{~cm}$ defects with sharp dissection to include the peritoneum and muscle layer on the opposite right and left sides of the abdominal wall. In group I (Control), the abdominal wall was closed with 4-0 PP suture (Prolene ${ }^{\circledR}$; Ethicon, Inc., Cincinnati, $\mathrm{OH}$, USA). In group II, a $3 \times 2 \mathrm{~cm}$ defect on the abdominal wall was created and a $4 \times 3 \mathrm{~cm}$ of PP mesh was used to repair the defect by suturing with 4-0 PP suture. In group III, the same procedure was performed as in group II. An additional sheet of H-CMC was layered between the PP mesh and abdominal organs. In group IV, PTFE mesh was used instead of PP; otherwise the same procedure was repeated as well as in group II. Composix ${ }^{\mathrm{TM}}$ mesh is constructed of two layers of low porosity expanded PTFE (e-PTFE). In group V, PET was implanted during the repair.

After 14 days, all rats were sacrificed under ether anesthesia via cervical dislocation and blood samples were taken. The mesh on the abdominal wall of the sacrificed rats in groups II-IV and group V were extracted as blocks with the surrounding fascial tissue. Strength of mesh incorporation was evaluated in grams by using a hand weighing machine scale and peak force required for disruption of the mesh from the abdominal wall. The breaking strength of the prosthetic material from the fascia was recorded as $\mathrm{g} / \mathrm{cm}^{2}$. The blocks containing fascia and the mesh were fixed buffered formalin solution and embedded into paraffin. Paraffin blocks were cut in 4 micron thick slices, stained with hematoxylin and eosin (H\&E), and examined under light microscope. Samples were evaluated with semi-quantitative scoring index in four degrees ( 0 , nil; 1 , minimal; 2 , moderate; 3 , more) with regard to giant cell, micro-abscesses, lymphocytes and plasma cells, fibroblastic activity and vascularization by blinded pathologist.

Additionally the fascial samples of $2 \times 0.5 \mathrm{~cm}$ were taken from the wound edge and hydroxyproline values were analyzed by the Switzer method (6) in the Department of Biochemistry of Istanbul University.

Statistical analysis. The hydroxyproline and breaking strength values between the groups were compared with one-way variance analysis (ANOVA post hoc Tukey's HSD analysis). The fibroblastic activity and vascularization as ordinal data were statistically evaluated by using Kruskal-Wallis with Bonferroni corrected Mann-Whitney U multiple comparisons, and Chi-square tests. Significance degree was accepted as $\mathrm{P}<0.05$. SPSS 19.0 software (SPSS, Inc., Chicago, IL, USA) was used for statistical analysis.

\section{Results}

All rats survived during the study period. The breaking strength was higher in group's II and III than in group IV and group V (Table I). The hydroxyproline level in group I was significantly higher than other groups and the lowest was in group V. Statistical analysis in group I compared to groups II-V showed significant values of $\mathrm{P}<0.01, \mathrm{P}<0.001, \mathrm{P}<0.0001, \mathrm{P}<0.0001$, respectively. Also group II had a statistically significant difference from group IV. Group V was different than other groups as shown in Tables I and II.
The average values obtained by scoring fibroblastic activity were the lowest in group V and the highest in group III (Fig. 1). Vascularization was the lowest in group IV and comparisons of the groups according to fibroblastic activity and vascularization can be seen in Table II. Giant cells in granulation tissue were seen only in the PP mesh-treated group. Also vascularization was increased in the PET group (Fig. 2). Abscess formation was more frequent in group IV than others. There was no statistically significant difference between groups with respect to either fatty necrosis in the fascia or inflammatory markers such as CRP and fibrinogen values in serum.

\section{Discussion}

There are both local and general factors that affect wound healing. Local factors are blood flow, presence of hematoma, infection, radiation, and tissue type. General factors which are related to the patient consists of anemia, anti-inflammatory and cytotoxic drugs, vitamin deficiencies, diabetes mellitus, uremia, jaundice, and infection (7-9). Experimental and clinical studies have shown that abdominal wall defects repaired with prosthetic materials give better results in tissue resistance tests compared to primary repair. These tests consist of breaking strength and histo-pathologic evaluations $(9,10)$. The pertinent criteria for ideal implantable biomaterial have been defined by Hamer-Hodges and Scott (11). The material should be chemically inert, non-carcinogenic, and capable of resisting mechanical strains, not excited by an inflammatory or foreign body reaction. But it is unlikely that a single 'ideal' prosthesis could fit all these properties $(3,12)$. The effect of prosthetic materials used in hernia repair on wound healing differs according to properties of the material. Frequently PP mesh plus H-CMC, PET and PTFE meshes are used in the repair of large abdominal wall defects. These were found successful in preventing adhesions. PET may be preferred if there is low fibroblastic activity and accumulation of hydroxyproline in direct bowel-associated with fascial defects.

The use of prosthetic material is recommended and PP mesh is the most commonly used material $(13,14)$. Propylene mesh may be preferred for hernia repair where high density, adhesion and breaking strength are required. However, its adhesive property causes intestinal and intra-abdominal adhesions. Sometimes, these are abnormal attachments, and cause significant morbidity and mortality after surgical procedures. The use of both H-CMC PTFE mesh and-PET mesh and were found to be equally effective in incisional hernia repair while decreasing intra-abdominal adhesions. It is also presented that wound healing may be affected with the use of prosthetic materials and anti-adhesive agents $(15,16)$. In order to reduce intra-abdominal adhesions and increase the abdominal tissue support, various prosthetic materials were used. Although PTFE meshes cause minimal intra-abdominal adhesions according to their structure, there are not any significant studies about mesh materials comparing their adhesion influences and breaking strengths (17). Dacron ${ }^{\circledR}$ was produced by both wall support material polyethylene terephthalate and non-absorbable adhesion barrier polyurethane. Adhesion barriers will prevent the postoperative intra-abdominal adhesions due to their non-absorbable properties (18). 
Table I. Results of the groups value according to fibroblastic activity, vascularization, breaking strength and hydroxyproline level.

\begin{tabular}{lcccc}
\hline Groups & $\begin{array}{c}\text { Fibroblastic } \\
\text { activity } \pm \text { SD }\end{array}$ & $\begin{array}{c}\text { Vascularization } \\
\pm \mathrm{SD}\end{array}$ & $\begin{array}{c}\text { Breaking strength } \\
\left(\mathrm{g} / \mathrm{cm}^{2}\right) \pm \mathrm{SD}\end{array}$ & $\begin{array}{c}\text { Hydroxyproline } \\
(\mu \mathrm{mol} / \mathrm{g}) \pm \mathrm{SD}\end{array}$ \\
\hline I & - & - & - & $49.52 \pm 6.5$ \\
II & $2.50 \pm 0.52$ & $1.92 \pm 0.29$ & $422.58 \pm 113.93$ & $36.10 \pm 9.9$ \\
III & $2.67 \pm 0.89$ & $2.33 \pm 0.89$ & $420.08 \pm 113.89$ & $35.22 \pm 7.5$ \\
IV & $1.50 \pm 0.80$ & $0.67 \pm 0.78$ & $182.42 \pm 85.32$ & $29.37 \pm 6.5$ \\
V & $1.00 \pm 1.27$ & $2.33 \pm 1.30$ & $198.21 \pm 62.03$ & $13.25 \pm 16.03$ \\
\hline
\end{tabular}

$\mathrm{SD}$, standard deviaition.

Table II. Comparisons of the groups according to fibroblastic activity, vascularization, breaking strength and hydroxyproline level.

\begin{tabular}{lcccc}
\hline Groups & Fibroblastic activity $^{\mathrm{a}}$ & Vascularization $^{\mathrm{a}}$ & Breaking strength $^{\mathrm{b}}$ & Hydroxyproline $^{\mathrm{b}, \mathrm{d}}$ \\
\hline II-III & $\mathrm{NS}$ & $\mathrm{NS}$ & $\mathrm{NS}$ & $\mathrm{NS}$ \\
II-IV & 0.005 & $<0.001$ & $<0.0001$ & $\mathrm{NS}$ \\
II-V & $0.008^{\mathrm{c}}$ & $\mathrm{NS}$ & $<0.0001$ & $<0.001$ \\
III-IV & 0.006 & $<0.001$ & $<0.0001$ & $\mathrm{NS}$ \\
III-V & $0.008^{\mathrm{c}}$ & $\mathrm{NS}$ & $<0.0001$ & $<0.001$ \\
IV-V & $\mathrm{NS}$ & 0.002 & $\mathrm{NS}$ & $<0.001$ \\
\hline
\end{tabular}

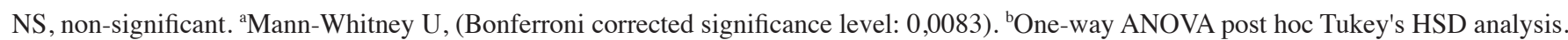
${ }^{\mathrm{c}} \mathrm{Nearly}$ significant. ${ }^{\mathrm{d}} \mathrm{Hydroxy}$ roline level in group I was significantly higher than other groups.

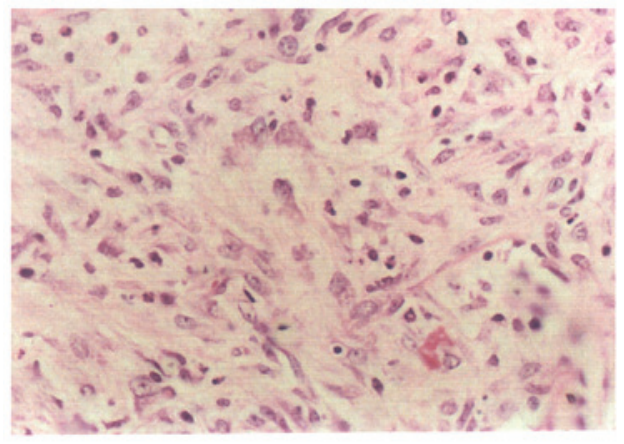

Figure 1. Granulation tissue in polypropylene and hyaluronate and carboxymethylcellulose-treated group. Note increased fibroblastic activity in the vicinity of the graft (hematoxylin and eosin, magnification, $\mathrm{x} 40$ ).

In this study our aim was to determine the influence of the PP-one layer mesh and dual mesh in wound healing and intra-abdominal adhesions. Also this experimental study has benefits that can be used in clinical attempts. PTFE and dual meshes were used in wide abdominal wall defects, laparoscopic incisional hernia repairs and in the case of contact with the mesh to the intra-abdominal organs (19). Also they can be used in infected wounds, tendency to infection, and stretched fascia avoids the relaxation. However, in the cases of tissue tension and collagen density they were fewer, the recurrence rates can increase in the same conditions (20). Our study has

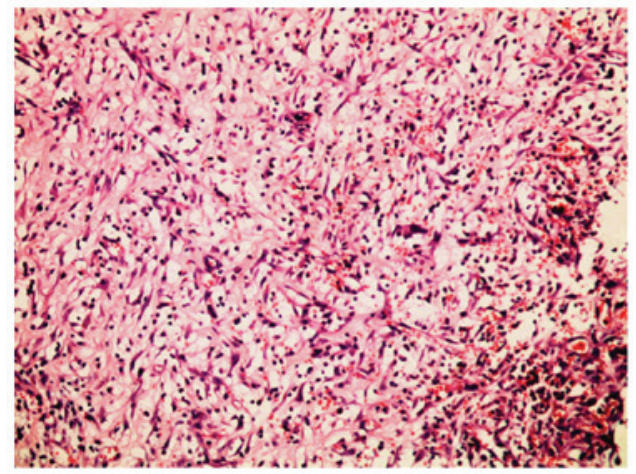

Figure 2. Increased vascularization in polyethylene terephthalate group (hematoxylin and eosin, magnification, $\mathrm{x} 20$ ).

shown that PET and PTFE mesh had less fibroblastic activity and hydroxyproline accumulation. It was also easy to break comparing other mesh groups.

On the other hand we want to evaluate the impact of the use of PP mesh with adhesion preventing materials instead of dual mesh. Many studies showed that the membrane formed of $\mathrm{H}-\mathrm{CMC}$ decreases the diffusion, incidence and severity of adhesions. H-CMC separates the tissues that can form adhesions in the normal wound healing process by acting as a temporary barrier. The effect of $\mathrm{H}-\mathrm{CMC}$ on wound healing is still controversial. Some authors demonstrated that 
$\mathrm{H}-\mathrm{CMC}$ has an adverse effect on wound healing and others have not (17,21-23). Dinsmore and Calton compared PP mesh $\mathrm{PP}$ mesh+H-CMC in rabbits. They notified that $\mathrm{H}-\mathrm{CMC}$ decreased the severity and diffusion of adhesions but didn't cause a decrease in the adherence power. Furthermore, there was no difference in the force of detachment from wall between PP mesh and PP mesh plus H-CMC groups (22). Becker et al concluded that H-CMC decreased the severity, diffusion and incidence of postoperative abdominal adhesions in their prospective double-blind randomized study (21).

We found that the breaking strength of the mesh from fascia in PP mesh and PP mesh plus H-CMC groups was much higher than the PET and PTFE mesh group. Hydroxyproline levels were found to be lower in experimental groups compared with the control group. Furthermore, there was a statistically significant decrease between the PP mesh group and PET and PTFE mesh group in respect to hydroxyproline levels. Although there is not any significant difference in PTFE and PET mesh groups for the intra-abdominal adhesions, evaluation of the breaking strength decreased in PTFE and PET mesh group compared to the PP mesh and PP mesh plus H-CMC groups. Also giant cells were detected only in the PP mesh group which is a clue of foreign body reaction. In an area where adhesion is expected, e.g., those that come into direct contact with the intestines, PP mesh+H-CMC may be preferred.

The PTFE group evoked less inflammation based on the findings with histo-pathologic index, such as fibroblastic activity and vascularization were less than the other two groups. PTFE mesh and PET mesh have similar properties for both intra-abdominal adhesions and breaking strength. Vascularization's were lower in the PTFE mesh group compared to the PET group, on the other hand hydroxyproline levels were lower in the PET mesh group. These results had differences compared to the PP mesh group for healing parameters. Both PET and PTFE mesh can be preferred for the treatment of hernias considering the price advantage and patients clinical condition.

Our study which compared four different meshes has shown that, PP mesh and PP mesh+H-CMC had a better breaking strength and better histopathologic indices than PET and PTFE mesh. PET or PTFE, which has low risk of adhesion, ie low fibroblastic activity, is preferred in hernia with tissue defects. For those exposed to high intra-abdominal pressures, the PP mesh, which forms intact tissue with effective fibroblastic activity, high content of hydroxyproline and breaking strength, may be preferred, given the low cost. Also hydroxyproline values were the lowest in PET. As a result PET and PTFE mesh were more fragile and slower in wound healing compared to PP mesh and PP mesh plus H-CMC. On the other hand, the features that seem unfavorable for wound healing avoid intra-abdominal adhesions.

\section{References}

1. Mullassery D, Pedersen A, Robb A and Smith N: Incisional hernia in pediatric surgery-experience at single UK tertiary centre. J Pediatr Surg 51: 1791-1794, 2016.
2. Duffy AJ, Hogle NJ, LaPerle KM and Fowler DL: Comparison of two composite meshes using two fixation devices in a porcine laparoscopic ventral hernia repair model. Hernia 8: 358-364, 2004.

3. Andersen LP, Klein M, Gögenur I and Rosenberg J: Long-term recurrence and complication rates after incisional hernia repair with the open onlay technique. BMC Surg 9: 6, 2009.

4. Phillips JD and Nagle AP: Minimally invasive approaches to incisional hernia repairs. J Long Term Eff Med Implants 20: 117-128, 2010.

5. Borrazzo EC, Belmont MF, Boffa D and Fowler DL: Effect of prosthetic material on adhesion formation after laparoscopic ventral hernia repair in a porcine model. Hernia 8: 108-112, 2004.

6. Switzer BR and Summer GK: Improved method for hydroxyproline analysis in tissue hydrolyzates. Anal Biochem 39: 487-491, 1971.

7. Walming S, Angenete E, Block M, Bock D, Gessler B and Haglind E: Retrospective review of risk factors for surgical wound dehiscence and incisional hernia. BMC Surg 17: 19, 2017.

8. Ryan TJ: Infection following soft tissue injury: Its role in wound healing. Curr Opin Infect Dis 20: 124-128, 2007.

9. Mäkelä JT, Kiviniemi H, Juvonen T and Laitinen S: Factors influencing wound dehiscence after midline laparotomy. Am J Surg 170: 387-390, 1995.

10. Franklin RR: Reduction of ovarian adhesions by the use of Interceed. Ovarian Adhesion Study Group. Obstet Gynecol 86: 335-340, 1995.

11. Hamer-Hodges DW and Scott NB: Surgeon's workshop. Replacement of an abdominal wall defect using expanded PTFE sheet (Gore-tex). J R Coll Surg Edinb 30: 65-67, 1985.

12. DeBord JR: The historical development of prosthetics in hernia surgery. Surg Clin North Am 78: 973-1006, vi, 1998.

13. Dinsmore RC, Calton WC Jr, Harvey SB and Blaney MW: Prevention of adhesions to polypropylene mesh in a traumatized bowel model. J Am Coll Surg 191: 131-136, 2000.

14. Alimoglu O, Akcakaya A, Sahin M, Unlu Y, Ozkan OV, Sanli E and Eryilmaz R: Prevention of adhesion formations following repair of abdominal wall defects with prosthetic materials (an experimental study). Hepatogastroenterology 50: 725-728, 2003.

15. Yelimlieş B, Alponat A, Cubukçu A, Kuru M, Oz S, Erçin C and Gönüllü N: Carboxymethylcellulose coated on visceral face of polypropylene mesh prevents adhesion without impairing wound healing in incisional hernia model in rats. Hernia 7: 130-133, 2003.

16. Di Vita G, Patti R, D'Agostino P, Ferlazzo V, Angileri M, Sieli G, Buscemi S, Caruso G, Arcara M and Cillari E: Modifications in the production of cytokines and growth factors in drainage fluids following mesh implantation after incisional hernia repair. Am J Surg 191: 785-790, 2006.

17. Chavarriaga LF, Lin E, Losken A, Cook MW, Jeansonne LO, White BC, Sweeney JF, Galloway JR and Davis SS Jr: Management of complex abdominal wall defects using acellular porcine dermal collagen. Am Surg 76: 96-100, 2010.

18. Abdollahi A, Maddah GH, Mehrabi BM, Jangjoo A, Forghani MN and Sharbaf N: Prosthetic incisional hernioplasty: Clinical experience with 354 cases. Hernia 14: 569-573, 2010.

19. Nardi MJ, Millo P, Brachet Contul R, Fabozzi M, Persico F, Roveroni M, Lale Murix E, Bocchia P, Lorusso R, Gatti A, et al: Laparoscopic incisional and ventral hernia repair (LIVHR) with PARIETEX $^{\mathrm{TM}}$ Composite mesh. Minim Invasive Ther Allied Technol 21: 173-180, 2012.

20. Qadri SJ, Khan M, Wani SN, Nazir SS and Rather A: Laparoscopic and open incisional hernia repair using polypropylene mesh-a comparative single centre study. Int J Surg 8: 479-483, 2010.

21. Becker JM, Dayton MT, Fazio VW, Beck DE, Stryker SJ, Wexner SD, Wolff BG, Roberts PL, Smith LE, Sweeney SA and Moore M: Prevention of postoperative abdominal adhesions by a sodium hyaluronate-based bioresorbable membrane: A prospective, randomized, double-blind multicenter study. J Am Coll Surg 183: 297-306, 1996.

22. Dinsmore RC and Calton WC Jr: Prevention of adhesions to polypropylene mesh in a rabbit model. Am Surg 65: 383-387, 1999.

23. Mueller PO, Harmon BG, Hay WP and Amoroso LM: Effect of carboxymethylcellulose and a hyaluronate-carboxymethylcellulose membrane on healing of intestinal anastomoses in horses. Am J Vet Res 61: 369-374, 2000. 